\title{
Adhesion controls bacterial actin polymerization-based movement
}

\author{
Frederick S. Soo*t and Julie A. Theriot ${ }^{\ddagger}$ \\ *Department of Physiology and Biophysics, University of Washington, Seattle, WA 98105; and ₹Department of Biochemistry, Stanford University, \\ Stanford, CA 94305
}

Edited by Thomas D. Pollard, Yale University, New Haven, CT, and approved September 19, 2005 (received for review August 14, 2005)

\begin{abstract}
As part of its infectious life cycle, the bacterial pathogen Listeria monocytogenes propels itself through the host-cell cytoplasm by triggering the polymerization of host-cell actin near the bacterial surface, harnessing the activity of several cytoskeletal proteins used during actin-based cell crawling. To distinguish among several classes of biophysical models of actin-based bacterial movement, we used a high-throughput tracking technique to record the movement of many individual bacteria during temperature shifts. The speed of each bacterium varied strongly with temperature, closely following the Arrhenius rate law. Among bacteria, the prefactor $\boldsymbol{A}$ of the Arrhenius dependence unexpectedly varied exponentially with apparent activation energy, $E_{a}$, over a wide range $(8-21 \mathrm{kcal} / \mathrm{mol})$, reminiscent of the "rate compensation effect" of classical catalytic reactions. Average $E_{\mathrm{a}}$ were increased for mutant bacteria deficient in binding Ena/VASP proteins and bacteria moving in diluted extract. These two effects were additive. The observed temperature and rate compensation effects are consistent with a class of simple kinetic models in which the bacterium advances through the thermally driven, cooperative breakage of groups of adhesive bonds on its surface. The estimated number of coupled adhesive bonds $N$ on the bacterial surface varies between 10 and $\mathbf{4 0}$ bonds. In contrast to other models, this model correctly predicts an experimentally observed negative correlation between bacterial speed and actin gel density. The idea that speed depends on adhesion, rather than polymerization, suggests several alternative mechanisms by which known cytoskeletal regulatory proteins could control cellular movement.
\end{abstract}

ActA | Listeria | motility

$\mathbf{M}$ any types of eukaryotic cells, including immune system lymphocytes, neurons, and cancer cells, use the forces generated by polymerizing actin filament networks to change shape and to move. In the actin polymerization-based movement of intracellular bacterial pathogens such as Listeria monocytogenes, individual bacteria harness the same cytoskeletal machinery to move within the host-cell cytoplasm (1).

Several classes of biophysical models have been proposed to explain the mechanism of actin polymerization-based movement. The models differ in which steps in the motility cycle are proposed to control the rate of bacterial movement.

The "tethered elastic Brownian ratchet" model (2) proposes that a large force generated by the network of polymerizing actin filaments in the "comet tail" behind the bacterium continuously induces the breakage of individual adhesive bonds between the bacterium and the surrounding actin gel, pushing the bacterium forward. In this model, bacteria move at the speed at which propulsive and adhesive forces are balanced; this equilibrium velocity depends strongly on the specific properties of actin polymerization and adhesion. The related "elastic gel" model (3) proposes that the elastic expansion of the actin gel behind the bacterium also contributes to propulsion, "squeezing" the bacterium forward.

The "actoclampin" model $(4,5)$ proposes that the adhesive bonds are never broken, and instead that the bacterium advances through the discrete, ATP-hydrolysis-driven advancement of "end-clamping" proteins that bind the bacterium to the elongating ends of actin filaments. In this case, the biochemical rates of hydrolysis and filament growth reactions determine the rate of movement.

Finally, a class of models, which we call "cooperative thermal breakage" models, propose that the bacterium advances through the rectified, thermally driven breakage of small groups of adhesive bonds. This class of models, which includes both a simple kinetic model, which we introduce here, and detailed computational models (6), differ from other models in that the strength of adhesion, rather than the rate of polymerization, is the dominant factor in determining the overall rate of movement.

The models make different predictions about how quickly bacteria should move at different temperatures, and how this temperature dependence should vary among bacteria. Under the simplest assumptions, the tethered elastic Brownian ratchet and elastic gel models predict that bacteria will move faster at higher temperatures, and also that the steepness of the temperature dependence will be related in a complex way to the characteristic average speed of that bacterium. In the actoclampin model, speed is strongly coupled to the rate of ATP hydrolysis (4), so the temperature dependence of all bacteria should follow that of ATP hydrolysis. The simple kinetic form of the cooperative thermal breakage model predicts that $(i)$ the speed of individual bacteria will increase approximately as an Arrhenius-type function of temperature and (ii) among bacteria, the temperature dependence will be "rate-compensated" $(7,8)$, i.e., the prefactor $A$ and the apparent activation energy, $E_{\mathrm{a}}$, of the Arrhenius dependence among individual bacteria will be correlated over a wide range.

To distinguish among these models, we used an automated high-resolution tracking system (9) to track a large population of individual bacteria as the temperature was varied slowly over the physiological range. Our results are presented in three sections. First, we present recordings of individual bacteria moving under temperature shift. The speed of individual bacteria increases dramatically with temperature, with a dependence well approximated by an Arrhenius-type function. Second, we show that the temperature dependence of a mutant strain of bacteria and bacteria moving in diluted extract is also well described by an Arrhenius-type relation. Finally, we present data collected from many bacteria. Among bacteria, the apparent activation energy and the prefactor $A$ of the Arrhenius relationship vary widely, with a strong positive correlation. The effects of mutation and dilution of the extract are independent and additive, and follow the same trend as natural variation among bacteria.

Our data lead us to favor the simple kinetic form of the cooperative thermal breakage model over other existing models. To account for our data, the tethered elastic Brownian ratchet,

Conflict of interest statement: No conflicts declared.

This paper was submitted directly (Track II) to the PNAS office.

†To whom correspondence should be addressed. E-mail: fsoo@u.washington.edu.

(C) 2005 by The National Academy of Sciences of the USA 
elastic gel, and actoclampin models must include new mechanisms to explain variation among bacteria, rate compensation, and sensitivity to genetic mutation and biochemical manipulation. These modifications add significant complexity to these models. The simple kinetic form of the cooperative thermal breakage model, in contrast, quantitatively explains many aspects of our data without modification, while relying on relatively few assumptions.

\section{Methods}

Bacterial Culture and Slide Preparation. Listeria monocytogenes strain DP-L4087 (10) were cultured overnight (9) in $10 \mathrm{ml}$ of Luria broth (LB) medium at $37^{\circ} \mathrm{C}$ with continuous agitation. An isogenic strain expressing the GRR variant of ActA lacking the polyproline II helices that bind Ena/VASP (11) was constructed and generously provided by Susanne Rafelski (Center for Cell Dynamics, University of Washington, Friday Harbor). Mutant bacteria were grown in LB with $10 \mu \mathrm{g} / \mathrm{ml}$ chloramphenicol. Wild-type and mutant bacteria were spun down, washed, and resuspended in with Xenopus buffer (12). Two microliters of bacterial suspension was added to $20 \mu \mathrm{l}$ of Xenopus egg cytoplasmic extract, along with $2 \mu$ l of tetramethylrhodamine-labeled actin (12), $2 \mu \mathrm{l}$ of ATP regenerating mixture, and $0.5 \mu \mathrm{l}$ of $1-\mu \mathrm{m}$-diameter latex beads (Polysciences). Diluted extracts used the same proportions, except that $10 \mu \mathrm{l}$ of extract was replaced with $10 \mu \mathrm{l}$ of Xenopus buffer. A small drop of suspension $(3 \mu \mathrm{l})$ was sandwiched between two $25-\mathrm{mm}$, \#2 thickness, round glass coverslips that had been cleaned with sulfuric acid, rinsed with distilled water, and dried in an $125^{\circ} \mathrm{C}$ oven overnight. The glass coverslips were incubated on the microscope stage for $15 \mathrm{~min}$ at room temperature before recording began.

Temperature Control and Video Microscopy. Water from a temperature-controlled bath was continuously circulated through a custom stage insert, and temperature was monitored by a digital thermocouple immersed in an insulating layer of water covering the slide surface. All images were taken by using a $\times 40$ oilimmersion objective (Nikon), mercury lamp, phase and epifluorescence optics, and a cooled charge-coupled device camera (Princeton Instruments) using METAMORPH software (Universal Imaging, Downingtown, PA). Phase-contrast and epifluorescence image pairs were taken at 2-sec intervals and saved for later processing. Individual bacteria could be tracked as long as they remained within the field of view and did not exceed speeds of $500 \mathrm{~nm} / \mathrm{sec}$, a threshold set by the tracking algorithm. The tracking accuracy of the system is $\approx 0.1 \mu \mathrm{m}$ as estimated from the relative movement of fixed latex spacer beads relative to one another (9).

Image Processing and Data Analysis. Custom image processing algorithms (9) were used to extract the position and orientation of phase-dense objects in the microscope field. To correct for image movement during temperature shifts, the average movement of latex beads in the field was subtracted from each frame of the image. The instantaneous speed was calculated by dividing the distance traveled between frames by the time between frames. The linear speed is the slope of the best-fit line to the regression of average displacement on time interval (9), removes overestimates of average speed due to high-frequency jitter in bacterial motion, and was calculated by using a 15 -sec sliding window. Instantaneous linear velocity as a function of temperature was fitted by using a least squares algorithm with confidence intervals generated from the variance of the linear velocity estimate.

Temperature dependence was determined by using a least squares fit of the Arrhenius function on linear speed as a function of temperature; error values are standard deviations of the fit calculated by using the variance of the measured speed data, which is dominated by physiological variation. Speed at 298 $\mathrm{K}$ was estimated from the fitted parameter values. The limits of measurement were calculated by determining, for a given activation energy, the fastest or slowest that a bacterium could be moving at $298 \mathrm{~K}$ and still remain within the instrumental speed limits at the highest $(323 \mathrm{~K})$ and lowest $(283 \mathrm{~K})$ temperatures attained.

Cooperative Breakage Model Fitting. The exact rate expression for the simple form of the cooperative thermal breakage model is $v=V_{0} 1 /\left(1+\operatorname{Exp}[-\Delta G / k T]^{-N}\right)$, where $V_{0}$ is a speed/time proportionality constant, and $1 /\left(1+\operatorname{Exp}[-\Delta G / k T]^{-N}\right)$ is the probability that a cooperative system with $N$ states and free energy difference $\Delta G$ occupies the highest energy state. For $\Delta G<k T$, this expression is well approximated to first order by $v \sim V_{0} \operatorname{Exp}[-\Delta G / k T]^{N}$, but for fitting purposes, the full nonlinear expression was used. The apparent activation energy at temperature $T$ is $E_{\mathrm{a}, T}=-k(\partial \ln [v] / \partial(1 / T))$, and the apparent prefactor is $A=v / \operatorname{Exp}\left[-E_{\mathrm{a}, T} / k T\right] . A$, as a function of $E_{\mathrm{a}}, V_{0}, E_{0}$, $S_{0}$, and $T$, is implicit in these definitions and can be evaluated numerically. The mean square difference between $A$ and the measured values of $A$ as a function of the measured $E_{\mathrm{a}}$ for all bacteria were minimized by using a standard nonlinear least squares minimization algorithm (IGOR PRO) with $V_{0}, E_{0}$, and $S_{0}$ as free parameters and a fixed temperature, $T$. Although there is a slight dependence of the apparent activation energy on the temperature, $T$, this dependence is insignificant across the physiological temperature range. Because our experimental data measures the average activation energy over the physiological range, we chose to use $T=298 \mathrm{~K}$ because it is close to the average temperature in our recordings; allowing $T$ to vary freely produced similar results.

\section{Results and Discussion}

Our first finding is that the speeds of individual bacteria increase dramatically as the temperature of the preparation is increased, with dependence well approximated by an Arrhenius-type function. We used a high-throughput tracking technique (9) to record the two-dimensional movement of individual bacteria as the temperature of the preparation was slowly varied between $10^{\circ} \mathrm{C}$ and $40^{\circ} \mathrm{C}$. A typical bacterial trajectory, superimposed on a single image from the time-lapse sequence (see Movie 1, which is published as supporting information on the PNAS web site), is shown in Fig. 1. The path curves with micrometer-scale persistence length throughout the recording (Fig. $1 A$ ). For this and other bacteria, the instantaneous speed (Fig. $1 B$ ) is strongly dependent on the temperature, $T$ (Fig. $1 C$ ) and can be closely approximated by an Arrhenius-type relation, $v[T]=A \operatorname{Exp}\left[-E_{\mathrm{a}} /\right.$ $k T$ ] (Fig. $1 B$ and $D$, red trace), where $E_{\mathrm{a}}$ is the apparent activation energy in $\mathrm{kcal} / \mathrm{mol}, A$ is a scaling factor with units of distance/time, and $k$ is Boltzmann's constant. For this particular bacterium, $E_{\mathrm{a}}=10.6 \pm 0.1 \mathrm{kcal} / \mathrm{mol}$, and $\ln [A]=15.0 \pm 0.1$. The average measurement uncertainty among wild-type bacteria was $\pm 0.25 \mathrm{kcal} / \mathrm{mol}$ and \pm 0.43 for $E_{\mathrm{a}}$ and $\ln [A]$, respectively. This uncertainty is larger than would be expected from the positional error in the tracking system alone (9) and appears to come from random, uncorrelated fluctuations in speed of over time. In this and other bacterial tracks, there was no evidence for hysteresis. The only bacteria poorly fit were those with obvious collisions with obstacles. These bacteria were excluded from further analysis.

The approximately Arrhenius-type shape of the temperature dependence of bacterial movement is surprising. The rate of actin-based movement of mammalian $3 \mathrm{~T} 3$ cells in culture peaks at $37^{\circ} \mathrm{C}$, falling off steeply for temperatures a few degrees higher or lower (13). The speed of motile fish keratocytes plateaus as temperature is increased (14). In actin-based bacterial motility, several biochemical factors that influence the speed of move- 
A
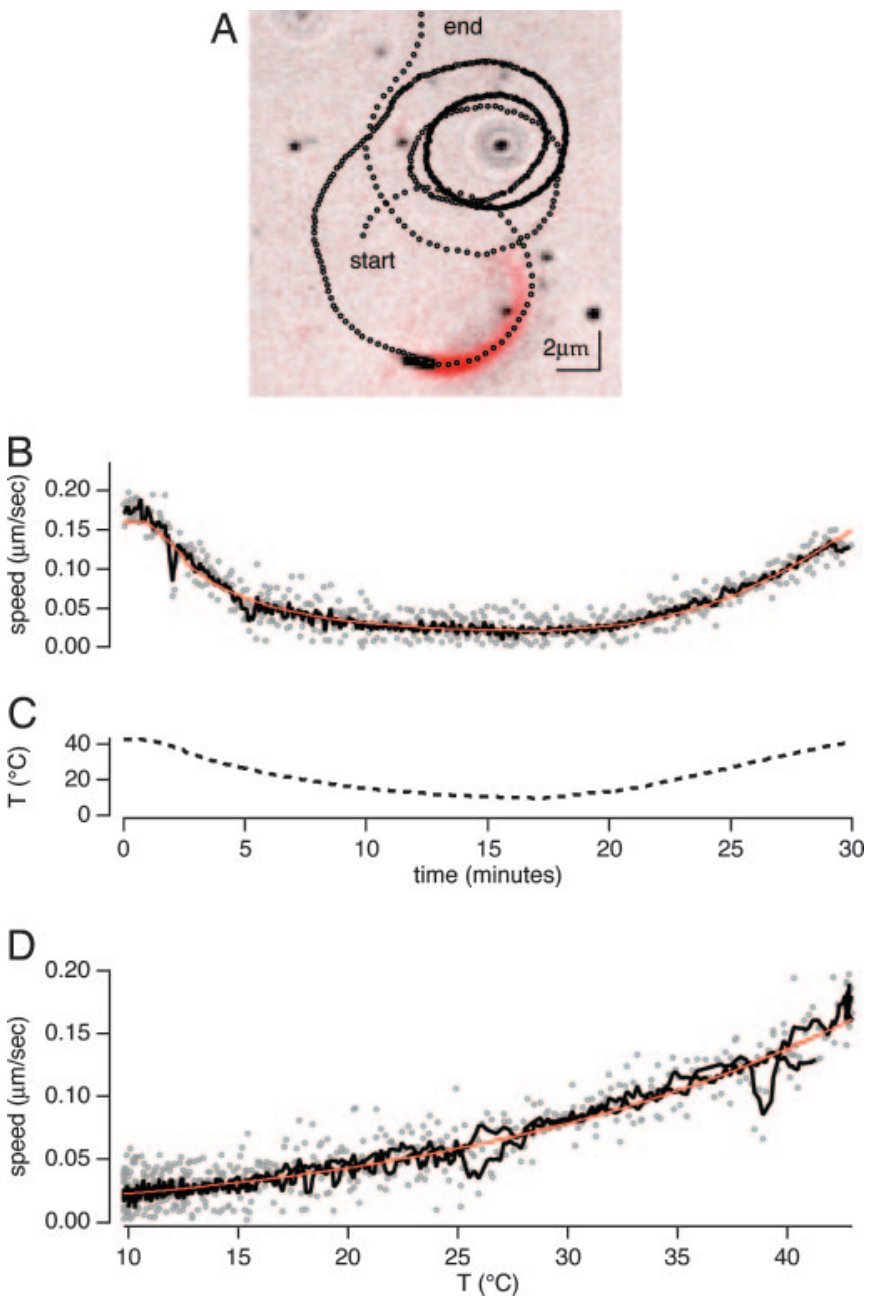

Fig. 1. Bacterial speed varies strongly with temperature. $(A)$ The twodimensional trajectory of the bacterium (black circles) is superimposed on a single composite phase and fluorescence image taken $120 \mathrm{sec}$ after the beginning of the recording. Each point represents the position of the bacterium at 2-sec intervals. $(B)$ The instantaneous speed (gray dots), linear speed (black line), and predicted speed using an Arrhenius relation (red line) are calculated as described in Methods and plotted as a function of time. (C) Bath temperature, $T$, as a function of time. $(D)$ Same data plotted as a function of temperature.

ment have been isolated and identified (15). Each could potentially contribute to the overall temperature dependence. We do not see a peak, a plateau, or a complex function. The simple temperature dependence that we observe is therefore either the product of several balanced processes or a single dominant process with Arrhenius-type temperature dependence.

Our second finding is that the Arrhenius-type temperature dependence is maintained even under biochemical and genetic manipulations that affect bacterial speed and the steepness of the temperature dependence. We tracked mutant bacteria expressing a mutated form of ActA that fails to bind the Ena/ VASP actin regulatory proteins $(11,16)$, wild-type bacteria in extract diluted with physiological buffer, which decreases the actin concentration, slowing the average bacterial speed (17), and mutant bacteria in diluted extract (Fig. 2). The twodimensional paths of individual bacteria appear to be similar under all four conditions (Fig. $2 A$ ), and the temperature dependence of bacteria under these conditions is still well described by an Arrhenius-type function (Fig. 2B). The individual mutant bacteria, wild-type bacteria in diluted extracts, and
A
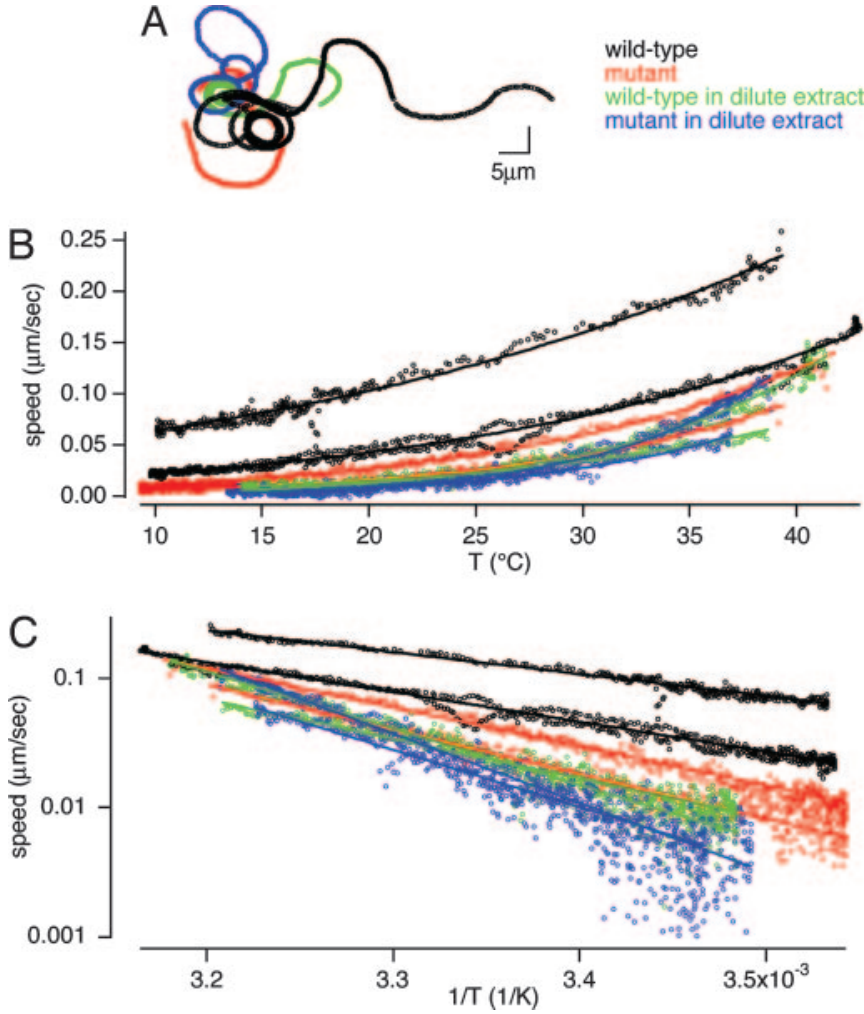

Fig. 2. Mutant bacteria and bacteria in diluted extract move more slowly and with stronger temperature dependence than wild-type bacteria. (A) Wildtype (black), mutant (red), wild-type in diluted extract (green), and mutant in diluted extract (blue) trajectories were translated and rotated so that they begin at a common origin and are headed initially to the right. The wild-type individual shown in Fig. 1 is one of the two wild-type examples. $(B)$ The linear speed as a function of temperature for each bacterium (colors as in $A$ ), along with the fitted speeds (lines) predicted from the bath temperature, $T$, using an Arrhenius relationship. (C) Same data shown as an Arrhenius plot.

mutant bacteria in diluted extracts tend to move more slowly than wild-type bacteria at room temperature, however, and with notably steeper temperature dependence. This is more clearly seen in Fig. 2C, where the same data are shown in an "Arrhenius plot" of $\ln$ [speed] vs. $1 / T$. In this format, Arrhenius-type temperature dependence appears as a straight line with a slope equal to $-E_{\mathrm{a}} / k$.

The third finding is that speed and apparent activation energy are correlated among all of the bacteria, whether wild-type or mutant, in full-strength or dilute extract. Across all four conditions, the speeds of individual bacteria at room temperature (298 $\mathrm{K})$ decrease systematically with apparent activation energy $E_{\mathrm{a}}$, as shown in Fig. 3B, where the speeds of all 288 bacteria are plotted as a function of $E_{\text {a }}$ (bottom axis). Each point represents a single bacterium, with error bars representing the standard deviation of the calculated uncertainty in the estimate of speed and $E_{\mathrm{a}}$. The average apparent $E_{\mathrm{a}}$ of mutant bacteria and wild-type bacteria in diluted extract is increased by $\approx 4 \mathrm{kcal} / \mathrm{mol}$ over wild-type (Fig. $3 A$, red and green), and the activation energy of mutants moving in diluted extract (Fig. $3 A$, blue) is increased by 9 $\mathrm{kcal} / \mathrm{mol}$ over wild-type (Fig. $3 A$, black). The speeds at room temperature decrease exponentially with $E_{\mathrm{a}}$ by a factor of 20 across all four conditions (Fig. $3 B$ ). The trend is not the result of limited sampling of a uniformly distributed population, because we did not see any slowly moving bacteria with shallow temperature dependence or rapidly moving bacteria with steep temperature dependence, even though these were well within the range of measurement of our tracking system. Like the bacte- 

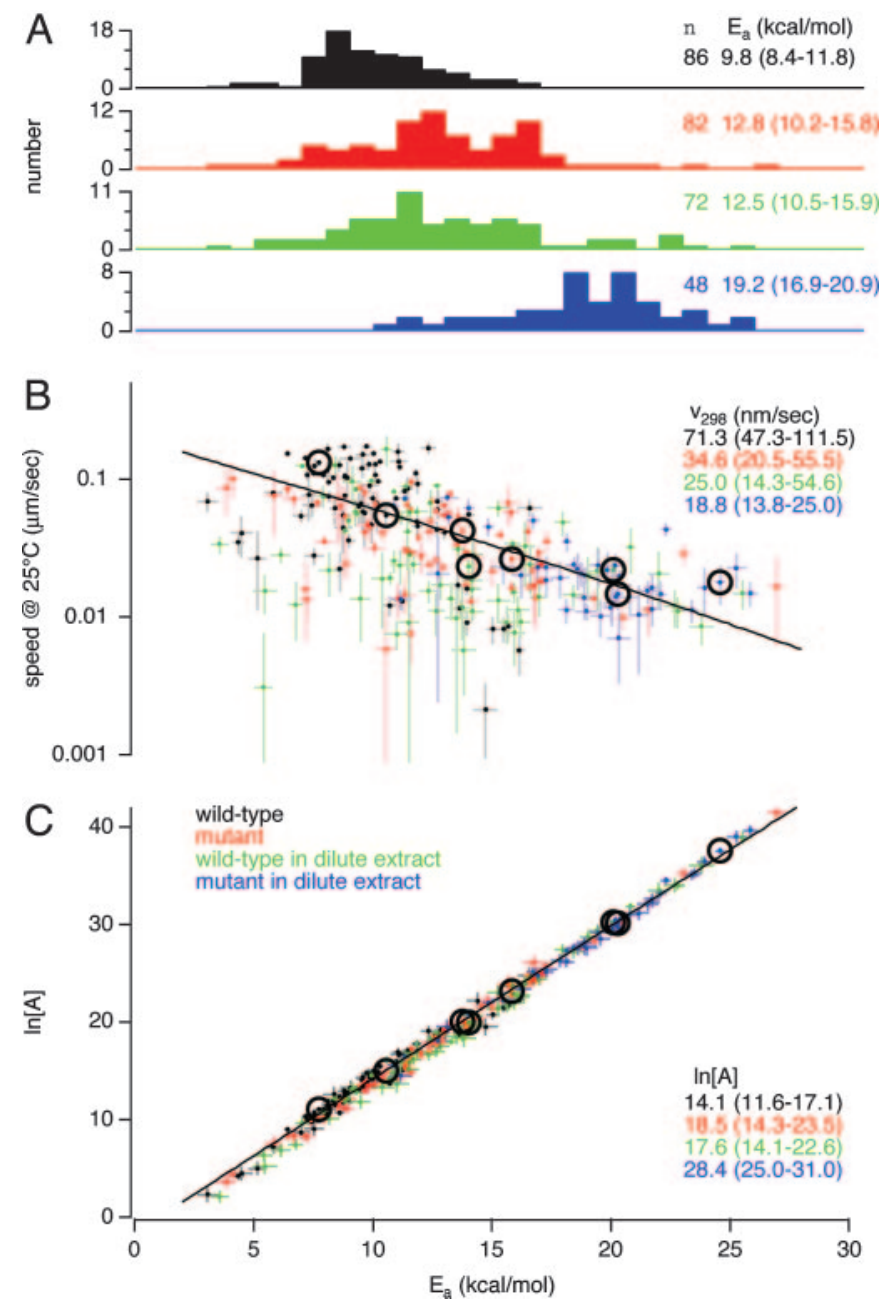

Fig. 3. Speed and $\ln [A]$ vary systematically with activation energy, $E_{\mathrm{a}}$. $(A)$ Histograms of $E_{\mathrm{a}}$ for wild-type (black), mutant (red), wild-type in diluted extract (green), and mutant in diluted extract (blue). Shown are sample size, $n$, median, $E_{\mathrm{a}}$, and intraquartile range. $(B)$ Individual rates of movement at 298 $\mathrm{K}$ as a function of $E_{\mathrm{a}}$. Error bars are standard deviations of estimate as calculated in Methods. Black circles indicate the eight examples shown in Fig. 2. The trend-line is from the least squares fit in $C$. The gray lines represent the limits of measurement (see Methods). (C) $\ln [A]$ as a function of $E_{\mathrm{a}}$. Colors, error bars, black circles, and measurement limits are as in $B$. The black line is the model least squares fit to all of the data.

rium shown in Fig. 1, the measurement uncertainties of individual bacteria, shown by the error bars, are small compared with the differences in $E_{\mathrm{a}}$ among bacteria, suggesting that differences in $E_{\mathrm{a}}$ originate from biological variation among the bacteria themselves rather than from imprecision in the measurement. The continuity of the trend across all four conditions suggests that the variation among all four conditions arises from a common source.

The direction of the trend indicates that the reaction mechanism is rate-compensated, i.e., while bacteria with high $E_{\mathrm{a}}$ move more slowly than those with low $E_{\mathrm{a}}$, they do not move nearly as slowly as would be predicted if only the activation energy differed between the two. As shown in Fig. $3 C, \ln [A]$ increases linearly with $E_{\mathrm{a}}$, with a linear relationship maintained over a range of seven orders of magnitude in $A$. Bacteria under all four experimental conditions follow approximately the same trend. This correlation compensates for what would otherwise be a drastic $10^{9}$-fold decrease in overall speed if $A$ remained constant over the same range of $E_{\mathrm{a}}$. A similar "rate compensation effect" is seen in many organic and inorganic reactions $(7,8)$. Because the rate compensation is not perfect, the bacteria still slow by a factor of 20 across the range of all activation energies (Fig. $3 B$ ), despite a factor of $\approx 10^{7}$ increase in $A$ over the same range. Again, the calculated measurement uncertainty for each bacterium, indicated by the error bars, is generally much smaller than the differences among bacteria, ruling out measurement uncertainty as a source of the trend. The degree of compensation is also too large to be accounted for by a change in step size, because the step size would have to change by a factor of $10^{7}$ and so must be due to an increase in the attempt rate of the reaction.

Our data place strong constraints on any physical model of movement. Any model must explain why each individual bacterium has a characteristic energy scale, $E_{\mathrm{a}}$, and speed scale, $A$, why these two scales are so closely linked among bacteria, and why genetic and biochemical manipulations change these scales along the same trend line as natural variations. If the temperature dependence is generated by a complex process, the model must explain how this process is compensated among bacteria to generate the precise correlation between $E_{\mathrm{a}}$ and $\ln [A]$ that we observe. If the temperature dependence is generated by a simple process, such as a single biochemical rate-limiting step, the model must explain how the biochemical activation energy of the rate-limiting step can vary among bacteria over a large range $(10-20 \mathrm{kcal} / \mathrm{mol})$, why the effects of mutation and dilution are independent and additive, and why the prefactor $A$ varies with the biochemical activation energy.

The existing forms of the tethered elastic Brownian ratchet and elastic gel can in principle account for the data, but only with extensive modification. Because they were originally designed to account for the movement of a single, canonical bacterium, a new mechanism must be added to account for the differences among bacteria that we observe. This mechanism must generate linear rate compensation over a wide range of activation energies and be sensitive to biochemical and genetic manipulations. Our calculations (described in Supporting Appendix, which is published as supporting information on the PNAS web site) suggest that for the tethered elastic Brownian ratchet model, such a mechanism would likely be highly complex, involving the correlated, nonlinear variation of several parameters among bacteria.

The actoclampin model can also account for the data, but only by invoking one of two possible explanations. The first is that all of the observed phenomena originate from the yet-unknown properties of the putative "end-clamping" protein, including the wide variation in $E_{\mathrm{a}}$ among bacteria, rate compensation, and sensitivity to mutation and biochemical manipulations. Although this hypothesis is not disproved by the data, there is also little evidence to support this view. Alternatively, the temperature and rate compensation effects could be due to individual differences in the number and arrangement of filaments among bacteria. This would run counter to the original motivation of this model, which was to explain movement in terms of the properties of a putative "end-clamping" protein, rather than the properties of filament organization and growth in the actin tail.

In contrast to the ad hoc modifications required to reconcile these models to our observations, our experimental results closely follow the predictions of the simple form of the cooperative thermal breakage model, which we define here. The model depends on three assumptions. First, the bacterium must behave as if it occupies two possible states, in which it is either "bound" or "unbound" to the surrounding actin gel, with relative probability dependent on the free energy difference between the states. Second, among bacteria, the enthalpic and entropic components of the free energy difference between the states must scale proportionally with one another. Finally, the rate of movement must be proportional to the probability that the bacterium occupies the unbound state. If these conditions are 
satisfied, it follows that $(i)$ individual bacteria should move with Arrhenius-type temperature dependence, and (ii) variations in activation energy among bacteria should be linearly ratecompensated.

Existing experimental evidence suggests that the three assumptions are satisfied during bacterial movement. The first assumption, that the energetic state of the bacterium is well approximated by a two-state model, is satisfied if, as suggested by existing experimental data, the bacterium is strongly attached to the surrounding actin gel (18) and does not move except when the attachment is broken, e.g., moves in discrete steps or intervals $(19,20)$. Under these conditions, at any moment in time the bacterium occupies one of two possible states, fixed and moving, with the energy difference between the states determined by the energy required to break the adhesion between the bacterium and surrounding actin gel. Assuming that the system is near thermal equilibrium and also that the time scale of the measurement is long compared with the transition time between states, the time-average probability of occupying either state then depends exponentially on the time-average free energy difference between the states. According to high-bandwidth laser interferometry measurements of bacterial motion $(19,20)$, the latter condition is true for the relatively long sample intervals that were used here $(2 \mathrm{sec})$.

The second assumption, that the total entropy and enthalpy scale proportionally among bacteria, is satisfied if multiple individual bonds must be simultaneously broken to free the bacterium. Again, existing measurements suggest that the bacterium is strongly attached to the surrounding actin gel by many adhesive bonds (18). In the simplest case where an average of $N$ adhesive bonds must be cooperatively broken to free the bacterium, and the free energies of individual bond breakage add linearly, the average free energy difference between the states is $N\left(\Delta H_{0}-T \Delta S_{0}\right)$, where $\Delta H_{0}$ is the enthalpy and $\Delta S_{0}$ the entropy of breakage of a single adhesive bond, and $N$ is the time-average number of adhesive bonds; in this case, the total enthalpy and entropy both scale linearly with $N$. A linear dependence of total energy on the number of bonds, $N$, is not an absolute requirement: any energy summation scheme will work as long as the total enthalpy and entropy scale proportionally. Because the system appears to spend most of its time in the bound state (19, $20)$, the probability $p$ that the bacterium occupies the unbound state is therefore approximately proportional to $\operatorname{Exp}\left[-N\left(\Delta H_{0}-\right.\right.$ $\left.\left.T \Delta S_{0}\right) / k T\right]$, where $k$ is Boltzmann's constant, and $T$ is the absolute temperature. This is an Arrhenius-type temperature dependence of the type we see in the movement of individual bacteria. If the bond energies sum linearly, $N$ is literally the average number of cooperatively breaking adhesive bonds, and in the nonlinear case, $N$ is the apparent number of bonds.

The third assumption, that speed be approximately proportional to the probability of occupying the unbound state, is satisfied if (as assumed by all existing models) the bacterium advances by a form of rectified thermal diffusion while unbound. In this case, the diffusion rate is expected to vary roughly as $T^{1 / 2}$ (21); for the biological range of temperatures (283-310 K), this is less than a $5 \%$ variation. In comparison, for a free energy difference of $3 \mathrm{kcal} / \mathrm{mol}$ the probability of being unbound is expected to increase by $\approx 1.5$-fold across the same range, and for a $20 \mathrm{kcal} / \mathrm{mol}$ apparent activation energy, more than a 20 -fold increase is expected. In either case, the energy required to break the bonds dominates the temperature dependence of movement, with only a minor contribution from the diffusive step. In a special case of the actoclampin model where ATP hydrolysis is accelerated so much that diffusion of the "end-clamp" protein becomes the rate-limiting step (4), the speed is expected to plateau and only increase as $T^{1 / 2}$ at higher temperatures.

It follows that the speed of individual bacteria, $v$, will depend on temperature with an Arrhenius-type relation $v=A \operatorname{Exp}\left[-E_{\mathrm{a}} /\right.$ $k T]$, where $E_{\mathrm{a}}=N \Delta H_{0}$ is the apparent activation energy, $A=V_{0}$ $\operatorname{Exp}\left[N \Delta S_{0} / k\right]$ is the prefactor, and $V_{0}$ is the speed proportionality constant $v=V_{0} p$. This prediction closely agrees with the individual Arrhenius-type dependences we observe in individual bacteria (Figs. $1 C$ and $2 B$ and $C$ ). Furthermore, because $\ln [A]=$ $\ln \left[V_{0}\right]+N \Delta S_{0} / k$, the model predicts that $\ln [A]$ and $E_{\mathrm{a}}$ should be approximately linearly correlated among bacteria with slope $\Delta S_{0} / k H_{0}$ and intercept $\ln \left[V_{0}\right]$. This prediction also agrees with the observed positive correlation between $E_{\mathrm{a}}$ and $\ln [A]$ among all bacteria, shown in Fig. $3 C$. Fitting the data with the exact model (see Methods and dark lines in Fig. $3 B$ and $C$ ) gives physiologically reasonable values of $V_{0}=0.41 \mu \mathrm{m} / \mathrm{sec}, \Delta H_{0}=$ $0.81 \mathrm{kcal} / \mathrm{mol}$, and $\Delta S_{0}=2.54 \times 10^{-3} \mathrm{kcal} / \mathrm{mol} \cdot \mathrm{K}=1.3 \mathrm{k}$. Fitting the subpopulations separately gave similar values (i.e., the fit to wild-type population gives $V_{0}=0.95 \mu \mathrm{m} / \mathrm{sec}, \Delta H_{0}=0.85$ $\mathrm{kcal} / \mathrm{mol}$, and $\left.\Delta S_{0}=2.46 \times 10^{-3} \mathrm{kcal} / \mathrm{mol} \cdot \mathrm{K}\right)$. Three parameters, rather than just two, can be determined from the data because the speed, $v$, of individual bacteria is known over a range of temperatures. That is, the population slope is approximately equal to the ratio $\Delta S_{0} / k \Delta H_{0}$, the population intercept is equal to $\ln \left[V_{0}\right]$, and only one combination of $\Delta S_{0} / k \Delta H_{0}$ can satisfy a given individual bacterial temperature dependence $v=V_{0}$ $\operatorname{Exp}\left[-N\left(\Delta H_{0}-T \Delta S_{0}\right) / k T\right]$ for constant $N$ and variable $T$.

Intuitively, the precise rate compensation among bacteria that we observe arises in this model because each individual adhesive bond contributes both enthalpic and entropic components to the overall free energy required to break the bacterium free from the surrounding actin gel. As the apparent average number of bonds $N$ binding the bacterium to the actin gel increases, the average thermal energy required to break the cooperatively acting group of bonds increases, but the entropic gain of breaking the bonds also increases, speeding the reaction and compensating for what would otherwise be a drastic decrease in reaction rate. If differences in the average number of cooperative adhesive bonds $N$ are the only differences among bacteria, it follows that $E_{\mathrm{a}}$ and $\ln [A]$ will co-vary linearly among bacteria, with $\ln [A]=E_{\mathrm{a}} \Delta S_{0} /$ $k H_{0}+\ln \left[V_{0}\right]$, as seen in our data. Such linear covariance between $\ln [A]$ and $E_{\mathrm{a}}$ has been long studied in classical chemical reactions and is commonly known as rate compensation $(7,8)$. The continuity of the trend among mutant bacteria and bacteria in dilute extracts suggests that these manipulations affect the same mechanism responsible for generating variation among wildtype bacteria, namely variation in the average apparent $N$ of individual bacteria.

The importance in the simple cooperative thermal breakage model of the apparent average number of bonds $N$ in determining bacterial speed has several implications. For wild-type bacteria, it follows that the median $n=E_{\mathrm{a}} / H_{0}$ is 14.2 bonds. This is consistent with estimates that several hundred filament ends are in close proximity to the bacterial surface (1). For wild-type bacteria, a small variation in average $N$ between 12.6 and 16.5 bonds causes an inverse exponential variation in average room temperature speeds between $112 \mathrm{~nm} / \mathrm{sec}$ and $47 \mathrm{~nm} / \mathrm{sec}$. Because small differences in bacterial speeds persist over minutes or longer (9), $N$ must also be precisely and robustly maintained over long periods of time. Whether this occurs through precise maintenance of $N$ from moment-to-moment or through the averaging of large moment-to-moment fluctuations in $N$ over time is not clear from our data, which are collected over relatively long, 2-sec intervals.

The sensitivity of bacterial movement to small changes in the apparent number of cooperative bonds $N$ suggests a simple mechanism by which a mutation in ActA that abrogates the binding of Ena/VASP proteins (11) slows movement and increases activation energy: Ena/VASP proteins normally enhance the rate of actin branch dissociation from the bacterial surface (16). Abolition of binding through mutation causes loss of this activity of Ena/VASP, increasing $N$ to 17.6 bonds 
on average, proportionally increasing $E_{\mathrm{a}}$, and halving the average speed. Many known cytoskeletal regulatory proteins, including the Ena/VASP family tested here, affect actin branching and polymerization. The high sensitivity of speed to small variations in the number of adhesive bonds suggests that their control of motility may not be through their effects on polymerization, as previously thought, but primarily through their effects on adhesion.

In addition, if the apparent number of adhesive bonds $N$ is directly proportional to the density of the actin tail near the surface of the bacterium, the simple form of the cooperative thermal breakage model predicts that the average speed of individual bacteria should be inversely and exponentially correlated with the average actin tail density. This is true for a large population of wild-type bacteria (9) (see Supporting Text and Fig. 4 , which are published as supporting information on the PNAS web site), as well as for the bacteria moving in diluted extracts, which have actin tails of higher density and also have higher $N$. This observation also contradicts the prediction by the elastic Brownian ratchet model that actin density and bacterial speed should be positively correlated or not correlated at all (2). Several mechanisms could generate a proportional dependence between $N$ and actin density, such as binding of actin filaments to the bacterial surface or interfilament binding in the actin gel.

The simple cooperative thermal breakage model also predicts that effects on the speed constant $V_{0}$ may be separable from effects on adhesive bond free energy. For instance, a change in the macroscopic extract viscosity may only affect $V_{0}$, so that the entire $\ln [A]$ vs. $E_{\mathrm{a}}$ trend-line will be shifted but the slope will remain unchanged. Conversely, a genetic manipulation which affects $\Delta H_{0}$ separately from $\Delta S_{0}$ should alter the slope, but not the offset of the trend-line.

Two features in the data are not well explained by the cooperative thermal breakage model. First, among all bacteria, there appears to be a lower limit to $E_{\mathrm{a}}$ near $7 \mathrm{kcal} / \mathrm{mol}$, above which the wild-type bacteria are clustered (Fig. $3 A$ ). It is possible that at low $N$, bacteria may simply detach completely from the surrounding actin gel without ever reattaching. Such freely diffusing bacteria are not tracked (see Methods). Second, the scatter about the rate-compensation trend line is much greater than can be attributed to measurement error (Fig. $3 B$ and $C$ ). Because of the scatter, it is difficult to resolve if the subpopulations corresponding to each condition fall on the same or slightly different trend lines. Neither the scatter about the trend-line nor the exact values of $V_{0}, \Delta H_{0}$, and $\Delta S_{0}$ under different conditions are predicted by the model. It may be possible that individual differences in shape or ActA surface distribution may modify $V_{0}, \Delta H_{0}$, and $\Delta S_{0}$ for individual bacteria.

The analytical model introduced here and the computational "nanosaltation" models described in ref. 6 are closely related. The three assumptions of the simple kinetic model, i.e., tight

1. Tilney, L. G. \& Portnoy, D. A. (1989) J. Cell Biol. 109, 1597-1608.

2. Mogilner, A. \& Oster, G. (2003) Biophys. J. 84, 1591-1605.

3. Gerbal, F., Chaikin, P., Rabin, Y. \& Prost, J. (2000) Biophys. J. 79, 2259-2275.

4. Dickinson, R. B. \& Purich, D. L. (2002) Biophys. J. 82, 605-617.

5. Dickinson, R. B., Caro, L. \& Purich, D. L. (2004) Biophys. J. 87, 2838-2854.

6. Alberts, J. B. \& Odell, G. M. (2004) PLoS Biol. 2, e412.

7. Bligaard, T., Honkala, K., Logadottir, A., Norskov, J., Dahl, S. \& Jacobsen, C. J. H. (2003) J. Phys. Chem. B 107, 9325-9331.

8. Leffler, J. F. (1955) J. Org. Chem. 20, 1202-1231.

9. Soo, F. S. \& Theriot, J. A. (2005) Biophys. J. 89, 703-723.

10. Lauer, P., Chow, M. Y., Loessner, M. J., Portnoy, D. A. \& Calendar, R. (2002) J. Bacteriol. 184, 4177-4186.

11. Auerbuch, V., Loureiro, J. J., Gertler, F. B., Theriot, J. A. \& Portnoy, D. A. (2003) Mol. Microbiol. 49, 1361-1375.

12. Theriot, J. \& Fung, D. C. (1997) in Cell Biology: A Laboratory Handbook, ed. Celis, J. E. (Academic, New York), 2nd Ed., pp. 157-175. binding of the bacterium to surrounding actin filaments, cooperative thermal breakage of adhesive bonds, and rectified diffusive forward movement, are either built into or observed in the computational models. If temperature effects are incorporated into the computational model, we expect that the simulated bacteria will also move with Arrhenius-type temperature dependence and linear rate compensation.

Many aspects of cooperative thermal breakage models are open to mechanistic interpretation. Although we suggest here that the adhesive bonds form between the bacterial surface and the surrounding actin gel [possibly mediated by an interaction between the bacterial ActA protein and the host cell Arp2/3 complex (22)], it is also possible that the relevant bonds are between actin filaments within the gel, and that bond breakage causes an internal rearrangement of the gel, allowing the bacterium to move forward a short distance. There also must be a process that prevents the bacterium from slipping backwards while it is in the free state. Whether this is slow expansion of the actin gel behind the bacterium or intercalation of actin monomer near the surface is unknown. The simple kinetic model predicts that as long as the temperature dependence of forward diffusion remains much smaller than that of the main bond breakage step, the exact mechanism of rectification should not affect the overall shape of the temperature dependence.

\section{Conclusion}

Our data closely agree with the predictions of a simple kinetic model based on cooperative thermal breakage of adhesive bonds, in which the rate of movement is determined by adhesion, and in which actin polymerization and ATP hydrolysis play a secondary role. Although the tethered elastic Brownian ratchet, elastic gel, and actoclampin models may be, in principle, modified to account for our data, these modifications are extensive and change the fundamental character of these models, substantially increasing their complexity with little gain in explanatory power. In comparison, cooperative thermal breakage closely and quantitatively accounts for the trends that we observe, yet depends on only a few, simple conditions. More importantly, the model succinctly and accurately describes the actual behavior of the system over a wide range of experimental conditions and makes several testable predictions. Individual bacteria move as if their speed is determined by the probability with which thermal motion disrupts the adhesive bonds between the bacterium and the surrounding actin gel.

We thank Susanne Rafelski and Matthew Footer (Stanford University) for providing mutant strains and technical support; Chip Asbury, Steve Block, Susanne Rafelski, Fred Rieke, and Julie Sneddon for their comments on the manuscript; and Dan Herschlag for helpful discussions. This work was supported by National Institutes of Health Grant AI36929 and a grant from the American Heart Association.

13. Thurston, G. \& Palcic, B. (1987) Cell Motil. Cytoskeleton 7, 361-367.

14. Ream, R. A., Theriot, J. A. \& Somero, G. N. (2003) J. Exp. Biol. 206, 4539-4551.

15. Loisel, T. P., Boujemaa, R., Pantaloni, D. \& Carlier, M. F. (1999) Nature 401, 613-616.

16. Samarin, S., Romero, S., Kocks, C., Didry, D., Pantaloni, D. \& Carlier, M. F. (2003) J. Cell Biol. 163, 131-142.

17. Cameron, L. A., Robbins, J. R., Footer, M. J. \& Theriot, J. A. (2004) Mol. Biol. Cell 15, 2312-2323.

18. Gerbal, F., Laurent, V., Ott, A., Carlier, M. F., Chaikin, P. \& Prost, J. (2000) Eur. Biophys. J. 29, 134-140.

19. Kuo, S. C. \& McGrath, J. L. (2000) Nature 407, 1026-1029.

20. McGrath, J. L., Eungdamrong, N. J., Fisher, C. I., Peng, F., Mahadevan, L., Mitchison, T. J. \& Kuo, S. C. (2003) Curr. Biol. 13, 329-332.

21. Berg, H. C. (1993) Random Walks in Biology (Princeton Univ. Press, Princeton).

22. Welch, M. D., Rosenblatt, J., Skoble, J., Portnoy, D. A. \& Mitchison, T. J. (1998) Science 281, 105-108. 